\title{
In Reply: "The importance of recognizing cannabinoid hyperemesis syndrome from synthetic marijuana use"
}

\author{
Cecilia J. Sorensen ${ }^{1} \cdot$ Kristen DeSanto $^{2} \cdot$ Laura Borgelt $^{3} \cdot$ Kristina T. Phillips $^{4}$. \\ Andrew A. Monte ${ }^{1,5,6}$
}

Received: 21 March 2017 / Accepted: 26 March 2017 /Published online: 5 April 2017

(C) American College of Medical Toxicology 2017

We thank Drs. Yoo, Liu, and Villamagna for reading our recently published article and appreciate their insights regarding the difficult nature of diagnosing cannabinoid hyperemesis syndrome (CHS) in patients using synthetic cannabinoids and the potentially large health burden of this illness.

Our systematic review identified the three referenced case reports of CHS [1-3] secondary to synthetic cannabinoid use and we included them in our synthesis. Although the clinical toxidromes associated with synthetic cannabinoids can differ from those of natural cannabinoids, synthetic cannabinoids interact with the CB1 receptor [4] and since these cases meet our criteria for CHS diagnosis [5], we believe that these cases should be labeled as CHS and treated as such.

Certainly, questioning about synthetic cannabinoids is warranted when CHS is suspected. Given that many patients do not consider synthetic cannabinoids a "drug," it may be prudent to query the use of all "herbal products" in a thorough evaluation

Cecilia J. Sorensen

Cecilia.Sorensen@denverem.org

1 Denver Health Residency in Emergency Medicine, Denver Health and Hospital Authority, Denver, CO 80204, USA

2 Health Sciences Library, University of Colorado Anschutz Medical Campus, Aurora, CO, USA

3 Skaggs School of Pharmacy and Pharmaceutical Sciences, University of Colorado Anschutz Medical Campus, Aurora, CO, USA

4 School of Psychological Sciences, University of Northern Colorado, Greeley, CO, USA

5 Department of Emergency Medicine, University of Colorado School of Medicine, Aurora, CO, USA

6 Rocky Mountain Poison \& Drug Center, Denver Health and Hospital Authority, Denver, CO, USA of cyclic vomiting in an otherwise well individual in whom other etiologies of cyclic vomiting have been ruled out.

It is unclear how many cases of CHS are attributable to synthetic versus naturally occurring cannabinoids. Clearly, synthetic cannabinoids are the fastest growing and most abused class of new psychoactive substances [6]. The failure to capture this drug class by traditional drug screens paired with the rising popularity should prompt clinicians to consider synthetic cannabinoids as a cause of CHS.

\section{Compliance with Ethical Standards}

Conflicts of Interest The authors declare that they have no conflicts of interest.

Sources of Funding Dr. Borgelt receives support from the Colorado Department of Public Health and Environment and the University of Colorado Skaggs School of Pharmacy and Pharmaceutical Sciences. Dr. Phillips receives support from NIH on Drug Abuse R15 DA041656 and R01 DA034957, and Dr. Monte receives support from NIH 1 K23 GM110516 and NIH CTSI UL1 TR001082.

\section{References}

1. Bick BL, Szostek JH, Mangan TF. Synthetic cannabinoid leading to cannabinoid hyperemesis syndrome. Mayo Clin Proc. 2014;89(8): $1168-9$

2. Ukaigwe A, Karmacharya P, Donato A. A gut gone to pot: a case of cannabinoid hyperemesis syndrome due to K2, a synthetic cannabinoid. Case Rep Emerg Med. 2014;2014:167098.

3. Hopkins CY, Gilchrist BL. A case of cannabinoid hyperemesis syndrome caused by synthetic cannabinoids. J Emerg Med. 2013;45(4):544-6.

4. Fattore L, Fratta W. Beyond THC: the new generation of cannabinoid designer drugs. Front Behav Neurosci. 2011;5:60.

5. Sorensen CJ, et al. Cannabinoid hyperemesis syndrome: diagnosis, pathophysiology, and treatment-a systematic review. J Med Toxicol. 2017;13(1):71-87.

6. Trecki J, Gerona RR, Schwartz MD. Synthetic cannabinoid-related illnesses and deaths. N Engl J Med. 2015;373(2):103-7. 